\title{
Return of individual research results and incidental findings in the clinical trials cooperative group setting
}

\author{
Michael Ferriere, $\mathrm{BS}^{1}$ and Brian Van Ness, $\mathrm{PhD}^{2}$
}

The National Cancer Institute (NCI)-funded cooperative group cancer clinical trial system develops experimental therapies and often collects samples from patients for correlative research. The cooperative group bank (CGB) system maintains biobanks with a current policy not to return research results to individuals. An online survey was created, and 10 directors of CGBs completed the surveys asking about understanding and attitudes in changing policies to consider return of incidental findings (IFs) and individual research results (IRRs) of health significance. The potential impact of the 10 consensus recommendations of Wolf et al. presented in this issue are examined. Reidentification of samples is often not problematic; however, changes to the current banking and clinical trial systems would require significant effort to fulfill an obligation of recontact of subjects. Additional resources, as well as a national advisory board would be required to standardize implementation.

Genet Med 2012:14(4):411-416

Key Words: biobank; clinical cooperative group; human subjects research ethics; incidental findings; research results

\section{INTRODUCTION}

This article will frame the cooperative group banks (CBGs) as an interesting case study in relation to the management of incidental findings (IFs) and individual research results (IRRs) in response to new recommendations made by Wolf et al. ${ }^{1}$ As previously described by Wolf et al., an IF is "a finding concerning an individual research participant ... that has potential health or reproductive importance and is discovered in the course of conducting research but is beyond the aims of the study." An IRR is "a finding concerning an individual contributor that has potential health or reproductive importance and is discovered in the course of research on the focal variables under study in meeting the stated aims of the research project."2 A brief history and discussion of the role of the CGBs in cancer research is provided. The basic structure of the CGBs in relation to the models described by Bemmels et al. ${ }^{3}$ are presented, relevant key processes and current policies are described, and some of the potential impacts of the 10 recommendations made in the Wolf et al. consensus paper ${ }^{1}$ are examined.

The National Cancer Institute (NCI)-funded cooperative group cancer clinical trials system has existed for more than 50 years. Currently, 10 US-based groups conduct clinical trials on experimental therapies for cancer in the United States and several other countries. As part of their research efforts, the cooperative groups have created and maintain biospecimen banks (i.e., the CGBs), the structure and governance of which are specific to each group.

The CGBs facilitate the collection of samples and conduct of translational studies for the cooperative groups. In addition to providing clinical information (e.g., demographics, disease- related tests, treatment, and outcome measures), trial participants submit biospecimens as a part of many clinical trial protocols. The biospecimens collected and banked are most often (cancer or surrounding normal) tissue and blood. In addition to providing clinical diagnostic information, these samples are often used for correlative research, such as developing predictive biomarkers. In some cases, residual samples remain after these studies are complete. In other cases, materials are collected per protocol without a defined end point but for unspecified future use. All banked materials are available for use in approved research, and such research has led to a wealth of scientific knowledge and improvements to patient care (http://cgb. cancer.gov/about/scientific.impact.html).

The clinical scopes of the groups vary, but they share a focus on large phase II and phase III cancer trials where many participating sites are needed to accrue the numbers of patients necessary to meet study end points. Over 3,100 sites in the United States and abroad participate in cooperative group trials, ${ }^{4}$ representing a wide range of sites potentially contributing specimens to the CGBs. Trial sample collections and associated research may occur throughout the time span of the trial. Particularly in phase III studies, where large numbers of patients are needed to provide statistical power for study objectives and where survival is a common primary end point, trial completion and data maturity may take several years. In many cases, research may be done on samples years after the completion of the trial. This length of time needed to complete trials and conduct research on samples may have an impact on the ability of the groups or CGBs to recontact participants about their results, or the impact on their health, and will be examined in the discussion.

${ }^{1}$ Eastern Cooperative Oncology Group Coordinating Center, Boston, Massachusetts, USA; ${ }^{2}$ Institute of Human Genetics, University of Minnesota, Minneapolis, Minnesota, USA. Correspondence: Brian Van Ness (vanne001@umn.edu)

Submitted 13 September 2011; accepted 6 January 2012; advance online publication 1 March 2012. doi:10.1038/gim.2012.14 
Most of the CGBs operate under an honest broker model for their banking activities. Biospecimens received from participating sites may or may not be accompanied by individually identifiable health information as defined by the Health Insurance Portability and Accountability Act (http://www.hipaa.com /2009/09/hipaa-protected-health-information-what-does-phiinclude/) in the form of initials or names. Received samples typically arrive with a patient code unique to the clinical trial. Although the CGBs remove protected health information and assign a unique code before transferring samples to approved researchers, most CBGs maintain a key linking the original identifiers (again, typically a protocol-related patient code) and the bank's code. This key is provided only to cooperative group operations office staff and statisticians responsible for association studies whereby the results of laboratory studies are associated with patient information such as clinical outcome on the cooperative group trial. Laboratory researchers work with the cooperative groups, rather than the CGBs, to access clinical data. Access to samples and associated data is regulated by the cooperative groups and the NCI, in addition to local institutional review boards (IRBs). However, because of the use of the honest broker model, and lack of reidentification, many projects on CGB specimens are not considered to be human subject research (per 45 C.F.R. 46.101).

The NCI Group Banking Committee (GBC) was formed in 2005. According to its charter "the purpose of the Group Banking Committee (GBC), is to improve the operation of the clinical oncology cooperative group human specimen banks and to coordinate banking activities among groups conducting phase III and large phase II clinical trials" (the charter is available from the GBC on request). Ten cancer clinical trial cooperative groups are GBC members. The GBC generates consensus documents, approving them by established voting processes. Approved documents are then implemented by the member groups. Groups may choose to use their own documents rather than those generated by the GBC but agree that the principles of the approved documents will be reflected in the local documents. Therefore, an examination of approved GBC documents is an expedient way to examine broadly representative policies and processes across the cooperative groups.

Informed consent templates employed by all cooperative groups have sections or stand-alone components devoted to tissue banking. These templates make patients aware that banked specimens would be available for future research, contingent upon their consent. In 2011, the GBC finalized updates of the banking consent template in an effort to ensure that important issues were clearly and consistently represented to patients across the group templates. Foremost among the issues was a uniform way to deal with and describe genetic studies, which had become an increasingly common requested use for banked specimens. The final template informs the patient that consenting to future use of supplied biospecimens allows these specimens to be used in studies involving inherited traits. The template contains the single, explicit, statement: "Research results will not be returned to you or your doctor." This language was developed as a GBC consensus and drafts have been submitted for review to a number of oversight bodies during development. Therefore, it would be correct to say that IRRs and IFs resulting from projects utilizing banked tissue are not currently returned. Deviations from this general policy may of course be made on a protocol-by-protocol basis. Return of IRRs that represent clinical trial end points (e.g., biomarker testing to determine clinical trial eligibility) would not be included in this general statement. Although the template wording illustrates current policy, it does not preclude change based on the Wolf et al. recommendations ${ }^{1}$ but would require a change in current policy.

The GBC material use agreement provides a template that outlines general cooperative group policies involved in the use of banked tissue, covering areas such as regulatory approvals, data use, and publication policies (http://cgb.cancer.gov/access/ forms.html). The GBC material use agreement, or a group-specific material use agreement that adheres to the same principles, must be signed by an investigator as a precondition of receiving CGB samples. Notably, the current document states investigators will not seek to reidentify subjects. Because investigators are restricted from obtaining patient identifiers or therefore attempting to contact them, the burden of returning IRRs and IFs in the current setting would fall to another entity in the CGB system. In light of the consent statement not to return results, this remains undefined.

Because the CGBs vary in structure and governance, as well as policies regarding IRRs and IFs, they likely differ in how they might adhere to the recommendations of Wolf et al. ${ }^{1}$ A survey of CGB directors was conducted by the authors with an aim of establishing some of the similarities and dissimilarities of general conduct of research and current opinions on treatment of IRRs and IFs in the cooperative group setting.

\section{METHODS}

A 29-item survey was created by the authors. Questions were formulated to accomplish two goals: (i) to gather data on the current policies of the CGBs, and (ii) to solicit initial impressions of CGB leadership on the ability of the CGBs to adopt the recommendations of Wolf et al. in terms of return of IFs and IRRs. A brief background was provided to respondents. Respondents were not provided with a draft of the Wolf et al. article. The survey was not intended for statistical analysis and was not created in consultation with a statistician or survey tool expert. It was administered online using Google Docs. Personal e-mail invitations were sent to all 13 directors of CGBs, representing 14 distinct CGBs. The initial invitation was sent to directors of banks known to the authors through the GBC and represented banks from nine GBC member groups. Ten surveys were completed, representing seven national cooperative groups. Personal e-mail follow-up requests for survey completion were made up to twice during the time span the survey was open. The survey was conducted under the assurance that results would be anonymous although names and CGB positions were collected to confirm which banks were represented in the results. As this did not involve any human subjects 
information, no IRB approval was necessary. No follow-up with responders was attempted after the completion of the survey.

\section{RESULTS}

The complete survey tool and results are available in the Supplementary Methods and Procedures online. A summary of results follows, presented in broad categories relevant to issues of returning IRRs and IFs.

\section{Types of specimens and data}

All 10 survey participants indicated that their bank houses samples such as tissue, blood, or sputum received directly from the institution that has collected the materials, as well as derived material, including DNA. Five responders indicated that their banks generate or receive genotype data whereas five did not.

\section{Patient identifiers}

Four responders indicated that their CGBs receive protected health information along with specimens; six did not. One responder indicated that a sample recipient might receive such protected health information along with samples, whereas the remaining nine indicated protected health information was not released. Similarly, nine replies indicated that researchers always received de-identified samples.

\section{Operational and regulatory structure}

Eight of those surveyed indicated "someone else in the cooperative group" was responsible for providing clinical data (such as outcome) associated with supplied specimens to researchers. One responder indicated that this was the responsibility of CGB. Similarly, eight stated that someone else in the cooperative group performed clinical associations, with two responders choosing the response "other."

Nine of 10 banks operate under an honest broker model. Eight indicated that they maintain a distinct IRB approval for their cooperative group-related banking operations and all 10 noted a requirement for investigators who receive specimens to obtain a distinct IRB approval at their own (as opposed to the bank's) institution.

\section{Current IF and IRR policy}

When asked whether there is an established method for disclosure of IFs and IRRs for the CGB, four responded that there was not, one noted the investigator was responsible, and two responses indicated that the cooperative group was responsible. Three submitted a response of "other."

Eight surveyed indicated that during the application process for banked specimens, there was no collection of a list of anticipated IFs or IRRs; of the other two responses, one indicated such collection was performed and the other chose "unknown."

Nine of 10 responders indicated that they receive information on IFs and IRRs from investigators using banked samples after their research is complete, whereas one indicated his/ her CGB did not. Seven stated their CGB had never returned
Table 1 Responses to "Does any consent form used as part of your Cooperative Group Banking function indicate that research results may be returned to patients of treating physicians if warranted for health reasons?"

\begin{tabular}{lc} 
Answer choices & Number of responses \\
\hline Yes & 1 \\
No & 3 \\
Sometimes/protocol dependent & 4 \\
Unknown & 2 \\
Other & 0 \\
\hline
\end{tabular}

Table 2 Opinions on entities that might be expected to return IFs and IRRs

\begin{tabular}{lcc} 
Answer choices & $\begin{array}{c}\text { Responses } \\
\text { regarding the } \\
\text { entity that should } \\
\text { be responsible for } \\
\text { return }\end{array}$ & $\begin{array}{c}\text { Responses } \\
\text { regarding the } \\
\text { entity that is best } \\
\text { positioned for } \\
\text { return }\end{array}$ \\
\hline Bank & 0 & 0 \\
Cooperative group & 4 & 4 \\
\hline Investigator & 1 & 2 \\
None of the above & 3 & 1 \\
should be responsible & 2 & 3 \\
\hline Other & & \\
\hline IF, incidental finding; IRR, individual research result. &
\end{tabular}

IFs or IRRs for a cooperative group study; two did not know if this has ever occurred. One responder chose the "other" response.

Five responders indicated that their CGB had a policy on return of IFs and IRRs whereas five did not have a policy. Regarding the presence of a policy within the bank's governing institution, six indicated that they did not know whether there was one, as it would not affect the CGB samples.

The answers to the question "Does any consent form used as part of your Cooperative Group Banking function indicate that research results may be returned to patients of treating physicians if warranted for health reasons?" were mixed and are presented in Table 1.

\section{Opinions}

Participants were asked what entities should be responsible for disclosure of IFs and IRRs, and which entities were best positioned for disclosure (Table 2).

Seven of those surveyed felt a requirement for CGBs to disclose IFs and IRRs would add costs to banking; two had no opinion, and one thought no costs would be added.

Of note, seven expressed interest in participating in the assembly of databases to allow comparison of approaches for the return of IFs and IRRs.

At the end of the survey, participants were asked to rate how much they agreed with six statements concerning return IFs and IRRs by the CGBs. Tallied results are provided in Table 3. 
Table 3 Responses to "How much do you agree with the following statement?"

\begin{tabular}{|c|c|c|c|c|c|}
\hline \multirow[b]{2}{*}{ Statements } & \multicolumn{5}{|c|}{ Number of responses per category } \\
\hline & $\begin{array}{c}1 \\
\text { (Completely } \\
\text { disagree) }\end{array}$ & 2 & 3 & 4 & $\begin{array}{c}5 \\
\text { (Completely } \\
\text { agree) }\end{array}$ \\
\hline $\begin{array}{l}\text { The CG Bank should be responsible for disclosure of IFs } \\
\text { and IRRs to patients }\end{array}$ & 10 & & & & \\
\hline $\begin{array}{l}\text { The CG Bank has access to perform CLIA-approved } \\
\text { validation testing of returned results where necessary }\end{array}$ & 4 & 3 & 1 & 1 & 1 \\
\hline The CG Bank is adequately funded to disclose IFs and IRRs & 9 & 1 & & & \\
\hline
\end{tabular}

CG, cooperative group; CLIA, Clinical Laboratory Improvement Amendments; IF, incidental finding; IRR, individual research result.

\section{DISCUSSION}

In this section, selected survey results are discussed with consideration of the 10 consensus recommendations of Wolf et al. ${ }^{1}$ in light of the background information presented, personal experience with the cooperative group system and the survey results. In general, bank directors recognized the potential benefits of returning IRRs and IFs but raised substantial concern over the complexities of implementation within the cooperative group setting.

The first recommendation of Wolf et al. is that “... Overarching responsibility for assuring that the biobank research system fulfills IF/IRR responsibilities should generally lie with the biobank."1 From this recommendation a number of logistical and administrative issues arise. In particular, recommendation 4 suggests that a central advisory board be created to work with bodies responsible for identifying and assessing IFs and IRRs, whereas recommendation 10 suggests that there is an important role of bank funders and regulators in the handling of IFs and IRRs. ${ }^{1}$ These types of recommendations will be complicated for the CGBs to implement by virtue of the cooperative group and CGB structure.

The cooperative groups are governed by central executive committees that determine policies and methods for implementation. As such, the biobank management does not have independent jurisdiction on handling samples or determining policies for recontact in the context of IFs and IRRs. Because the biobanks are a widely distributed resource, within the clinical trial operation as well as in distributing samples to multiple secondary researchers, governance responsibilities must be central to the cooperative group management structure that oversees bank operations.

Similarly, the creation of an oversight body such as a Return of Results Oversight Committee as recommended in Wolf et al. ${ }^{1}$ should be sensitive to the governance of the bank and include representatives of all stakeholders in the biobank. The creation of an expert, multidisciplinary, advisory committee or board would be unquestionably beneficial, but issues of governance, scope, and finance complicate the issue. Some CGBs may be housed in an institution where such a board already exists (e.g., the Mayo Clinic) in relation to one or more programs or projects. It is unlikely that such an existing board would already have governance over the bank. In our survey, seven banks indicated that the IF/IRR return policy of their governing institution would not apply to the CGB project. The decision of whether an independent or shared board needs to be created to oversee IFs and IRRs related to the CGBs should be decided jointly by each relevant cooperative group and governing institution, and a suitable relationship formed. The scope of a board, or boards, in deciding whether results should be returned, what results would be returned, and how they should be returned would be decided at this time. The cooperative group or its member institutions would be expected to be a party in dissemination of IFs and IRRs and may therefore require representation on the oversight board.

Maintaining an oversight board would represent an additional operating expense for the cooperative group or the CGB's governing institution. Because decisions would need to be archived indefinitely as well as made available to IRBs on a regular basis, additional administrative tasks are created. These duties would need to be accounted for in terms of training and percentage effort of some staff, within the CGB, the governing institution, or the cooperative group. The governing institution and cooperative group would need to agree on the financing, scope, makeup, etc., of such a group. Alternatively, a federal body with advisory capacity to all CGB could be explored.

The development and maintenance of a roster of IFs and IRRs by the CGBs as recommended ${ }^{1}$ represents a commendable idea. Creation and maintenance of a database for this roster would require funding. Assumption of this responsibility by a federal body, such as the NCI in the context of the CGBs, would relax the burden. A centrally maintained, Web-accessible database with a simple update system for adding new findings would be preferable to the development and maintenance of independent systems across the CGBs. The benefits to such a system include (i) reduction in overall costs by eliminating redundant system development; (ii) a stable, publicly funded environment for the database, and (iii) a wealth of information from participants across the 
$\mathrm{NCI}$ available to other patients and other interested users and researchers. Well-recognized organizations such as the NCI's GBC or Office of Biorepositories and Biospecimen Research should be asked to recommend specifics of such a system, including architectural advice facilitating future data sharing.

Other recommendations of Wolf et al. ${ }^{1}$ deal with the question of whether banks should even be set up to allow reidentification (i.e., recommendations 2 and 6) and when they are, whether there is a responsibility for recontact and other dissemination of research results (i.e., recommendations 7 and 9). For most cooperative banks, reidentification is technically possible in many cases, although logistically challenging in the current system. Re-linking of results with a cooperative group study and case identifier can often be done via coordination between the CGB and the cooperative group. This re-linking could potentially be extended to reidentification, assuming the ability and cooperation of the group in the endeavor. However, challenges are noted in the following.

The most recent information about a patient is housed elsewhere within the cooperative groups' operations offices, which may be distantly located from the CGB. For example, reports that a patient has died, moved, or changed treating institutions are sent to an operations office rather than to the CGB. Therefore, although the bank may have some information regarding identification of the patient, close coordination with the cooperative group data center would be required to identify a patient and his/her current status for follow-up, or relevant treatment site to contact. Yet the cooperative groups do not collect direct contact information for patients such as addresses, e-mails, or telephone numbers; they rely on the member sites to contact patients. The groups would need to either effect substantial changes in their policies and procedures in this regard (e.g., directly collect patient contact information) or work closely with participating sites to reidentify patients.

It should not be a difficult matter from an informatics standpoint to implement such changes prospectively. However, the idea of not being personally identified by research is central to the current tissue banking consent process and the impact of a change to this paradigm should be considered and studied. Fears of genetic results being used against a person when applying for employment or various types of insurance, whether well founded or not, may be a negative stimulus to patients to participate in tissue banking. Conversely, the idea of a possible warning against future health issues might be an enticement to others to participate. Given that only about $3 \%$ of adult patients participate in clinical trials (http://www.nap.edu/openbook. php?record_id=12879\&page=191), the pool of those supplying tissue to the system is small and policy changes must be carefully considered so as to create a positive impact or minimize negative impact on the goals of cooperative group clinical trials.

Alternative methods of reidentification exist, and should be explored. Aggregate results can and should be widely publicized, as suggested in recommendation 9 by Wolf et al. ${ }^{1}$ Results could be accompanied by invitations to former participants to initiate their own recontact. A drawback of this method is that not all patients would be reached in this way. However, a reversed recontact process would have the benefit of reaching those patients most interested in learning about results, and limit expenses in pursuing patients effectively lost to the followup process. Some resources, such as a study-specific recontact website, would be required but might be supplied by existing communication structures associated with the NCI Cooperative Group system. The Coalition of Cancer Cooperative Groups' website-http://www.cancertrialshelp.org-maintains a clearinghouse of cooperative group research-oriented press releases and resources for patients, advocates, and caregivers. The NCI's clinicaltrials.gov website operates under a mandate to provide access to trials and to provide the results of those trials in lay language. There are also patient advocacy and patient representative structures within the groups; these have proven to be energetic and effective in accessing the patient population. However, although these mechanisms can be used to disseminate aggregate results, interacting with individuals requires expert and readily available staff. This role is unlikely to be filled by researchers themselves or public relations staff who would be most likely to create the announcements and websites. Again, the issue of available resources to supply such staff arises. Based on the survey results, the CGB leadership polled did not feel they currently have the expertise or resources to most effectively return findings.

Another complicating factor in whether CGBs should be responsible for disclosing individual results is introduced by virtue of the patient having no clinical relationship with the bank. The CGB will rarely be at the treating institution, as there are a handful of CGBs and hundreds of participating institutions. Patients are not given much information about the CGB itself in the current consent template, which focuses on security safeguards and potential future testing of biospecimens. A recontacting role for the bank would probably require additional information in the consent form about bank personnel, their preferred methods of recontact, etc.

The lack of a relationship between CGBs and patients is an important consideration if a patient might expect further clinical explanations and options for action. Foremost, the bank has no information as to whether the condition has already manifested or testing has already been conducted for another reason. Addressing this issue would require dissemination of health-care information from one or more treating sites. In many instances, when research on banked tissue is conducted, the patient will have completed his/her involvement with the clinical trial and may no longer be in the care of a cooperative group-related physician. Therefore, neither the contributor nor his/her current caregiver may have a relationship with the CGB staff responsible for contacting them. This poses a difficulty in the CGB providing informed clinical referrals per recommendation 7 of Wolf et al., which suggests that biobanks should have plans in place to recontact contributors. ${ }^{1}$ Due to lack of familiarity with individual patients and the geographically disparate nature of the participating sites contributing to CGBs, providing a reasonable referral would be improbable in the majority of cases. 


\section{Conclusions}

Because of the need to track ongoing treatment outcomes, cooperative group clinical trials typically maintain identifiers at the site of trial treatment, so reidentification is often not problematic. However, changes to the system and increased effort would be required to fulfill an obligation of recontact of subjects on the part of the CGBs. Existing cooperative group policies and related documents would need to be updated to reflect and implement such a shift. Fundamental cooperative group processes, such as patient enrollment, consent, and follow-up would be significantly altered. The processes of application for use of banked materials require regulatory approvals, and material transfer agreements would need to be mindful of IRR and IF issues. Adequate resources for recontact would need to be in place, such as specialized training for responsible bank personnel or access to such individuals from other aspects of the bank's home institution.

Expected resource needs, both incremental and substantial, have been a common theme throughout the examination of the expected impact of the 10 recommendations of Wolf et al. ${ }^{1}$ Some central assistance by the NCI would alleviate some of the anticipated regulatory and administrative burden, but real impact on the CGBs in the actual recontact of patients would be unavoidable. Given their complicated organizational structures and importance based on the vast inventories of well-annotated, reidentifiable samples, the CGBs should be an important component of further discussion of handling IFs and IRRs in the modern research environment.

\section{SUPPLEMENTARY MATERIAL}

Supplementary material is linked to the online version of the paper at http://www.nature.com/gim

\section{ACKNOWLEDGMENTS}

This work was supported by National Institutes of Health/ National Human Genome Research Institute grant award no. 2-R01-HG003178-03 on "Managing incidental findings and research results in genomic biobanks \& archives" (principal investigator: S. Wolf). The authors thank the National Cancer Institute Group Banking Committee, particularly those individuals who completed the survey reported in this article. The authors are members of the Eastern Cooperative Oncology Group (ECOG) and thank the leadership of ECOG and ECOG's tissue banks for support and guidance. This study was coordinated by ECOG and supported in part by Public Health Service grants CA21115 and CA114737 (Robert L. Cornis, Principal Investigator), and grants from the National Cancer Institute, National Institutes of Health, and the Department of Health and Human Services. The contents and opinions expressed in this article are solely the responsibility of the authors and do not necessarily represent the official views of the National Cancer Institute.

\section{DISCLOSURE}

The authors declare no conflict of interest.

\section{REFERENCES}

1. Wolf SM, Crock, BN, Van Ness, B, et al. Managing incidental findings and research results in genomic research involving biobanks and archived data sets. Genet Med, 2012;14:361-384.

2. Wolf SM, Lawrenz FP, Nelson CA, et al. Managing incidental findings in human subjects research: analysis and recommendations. J Law Med Ethics 2008;36:219-248.

3. Bemmels, H., Wolfe, S, and Van Ness, B. Mapping the inputs, analyses, and outputs of biobank research systems to identify sources of incidental findings and individual research results for potential return to participants. Genet Med, 2012;14:385-392.

4. National Cancer Institute FactSheet, NCl's Clinical Trials Cooperative Group Program. http://www.cancer.gov/cancertopics/factsheet/NCl/clinical-trialscooperative-group. 